\title{
Transformando ações extensionistas para o ambiente digital: Um relato de experiência no projeto Meninas Digitais do Vale
}

\author{
Maria Victoria S. Fiori, Maria Elanne M. Rodrigues, Lauana Maria C. de \\ Oliveira, Ariany M. Almeida Maia, Marina da S. Rocha,Victoria Gabriella R. \\ Pereira, Jacilane de H. Rabelo, Anna Beatriz Marques \\ Universidade Federal do Ceará (Campus Russas) - Russas, CE - Brasil \\ \{victoria.fiori, elannemendes, lauanacartaxo, arianyalmeida, marinarocha, \\ victoriagabriella\}@alu.ufc.br, \{jacilane.rabelo, beatriz.marques\}@ufc.br
}

\begin{abstract}
The project Meninas Digitais do Vale project aims to strengthen female participation in the University's Computing courses Federal University of Ceará - Russas Campus. Due to the pandemic, caused by the new coronavirus COVID-19, social isolation and the adaptation of academic activities to the remote mode was necessary. In view of this, the project developed its actions remotely with the objective of continuing its extension activities during the period of social detachment, having social networks as its main ally. The project promoted long and short-term courses, production of thematic content in the area and events aimed at the academic community and the general public. To assess the experience of the participants in the actions, questionnaires were adopted. A quantitative and qualitative analysis was conducted on the results of the evaluation of the courses and actions promoted by the project.
\end{abstract}

Resumo. O projeto Meninas Digitais do Vale visa fortalecer a participação feminina nos cursos de Computação da Universidade Federal do Ceará Campus Russas. Devido à pandemia, ocasionada pelo novo coronavírus COVID-19, foi necessário o isolamento social e a adaptação de atividades acadêmicas para o modo remoto. Diante disso, o projeto desenvolveu suas ações de forma remota com o objetivo de continuar suas atividades de extensão no período de distanciamento social, tendo como principal aliada às redes sociais. $O$ projeto promoveu cursos de longa e curta duração, produção de conteúdos temáticos da área e eventos direcionados à comunidade acadêmica e ao público em geral. Para avaliar a experiência das participantes nas ações, foram adotados questionários. Uma análise quantitativa e qualitativa foi conduzida sobre os resultados da avaliação dos cursos e ações promovidas pelo projeto.

\section{Introdução}

O projeto Meninas Digitais do Vale, criado em 2018, tem como objetivo fortalecer a participação feminina nos cursos de Tecnologia da Informação (TI) da Universidade Federal do Ceará (UFC) - Campus Russas. O projeto realiza diversas ações voltadas ao público feminino dos cursos de Ciências da Computação e Engenharia de Software para engajamento, inclusão e troca de experiências entre meninas. Com o novo cenário acadêmico, devido à pandemia do COVID-19 decretada pelo Ministério da Saúde na Portaria $\mathrm{n}^{\circ} 188$, de 3 de fevereiro de 2020, buscou-se promover ações que fossem realizadas nessa nova realidade com a mesma qualidade da forma presencial. O público alvo dessas ações foi acessado através das redes sociais do Projeto Meninas Digitais do Vale na tentativa de atrair mais pessoas para as ações realizadas. 
De acordo com Nobre (2020), o ensino remoto conduz à reflexão, à valorização da escuta ativa e ao reconhecimento de que esta troca, esta partilha, não se rege somente pelo processo de ensino-aprendizagem. Estabelece-se, sobretudo, pelo ensino-partilha-aprendizagem. Maciel e Bim (2016) relatam a importância de realizar ações que visam fortalecer e incentivar a participação feminina e compartilhá-las com a sociedade para que assim os projetos possam atenuar o problema de ingresso de meninas no ensino superior. Logo, é importante desenvolver atividades que incentivem o desenvolvimento acadêmico, gerem conhecimento sobre a área computacional e compreendam as dificuldades enfrentadas no cenário atual.

O objetivo desse artigo é apresentar as principais ações e pesquisas realizadas pelas integrantes do projeto no formato remoto, relatando os resultados alcançados, desafios enfrentados devido ao cenário atual e lições aprendidas. Dessa forma, espera-se que tais ações sirvam de inspirações para os demais projetos parceiros do Programa Meninas Digitais. O restante desse artigo está dividido em seis seções: a Seção 2 apresenta o background e trabalhos relacionados. A Seção 3 relata as ações realizadas em ambiente digital. Na Seção 4 são discutidos os feedbacks sobre as ações realizadas. $\mathrm{Na}$ Seção 5 são apresentadas as lições aprendidas. Por fim, na seção 6 são expostas as conclusões e os trabalhos futuros.

\section{Background e Trabalhos Relacionados}

O projeto Meninas Digitais do Vale com intuito de se manter ativo e estimular outras mulheres realizou suas ações de forma online e utilizou das redes sociais para divulgação, assim conseguindo alcançar um público maior de participantes. Partindo disso, nesta seção serão apresentados trabalhos de projetos que objetivam diminuir a disparidade entre os gêneros, e buscam incentivar a atuação feminina na área da Computação, com ações realizadas antes e durante a pandemia.

O Projeto Meninas na Computação - UNIFAP (Universidade Federal do Amapá) comumente desenvolve ações, como oficinas, para a comunidade externa. Como mostra de Oliveira et al. (2020b), essas atividades foram modificadas para a forma remota e enfrentaram desafios na adaptação, pois houve uma grande procura pelas oficinas, impossibilitando atender a demanda, porém a repercussão das oficinas serviu para proporcionar a discussão sobre a presença de mulheres na Computação e na Ciência.

Segundo Frigo et al. (2020), o projeto Meninas Digitais-Regional Sul (UFSC) realizou um estudo sobre as oficinas voltadas para o ensino de algumas áreas de Computação com o objetivo de instigar a curiosidade de alunas do ensino público e privado nas áreas de pSTEM (physics, Science, Technology, Engineering and Mathematics). Foram coletados depoimentos de alunas participantes, que demonstraram o quanto as ações do projeto servem como incentivo para ingresso feminino em cursos na área da TI. A preocupação no baixo índice de ingresso e permanência de mulheres em cursos de Computação, fez com que o Projeto Garotas Tech dos Sertões de Crateús, promovesse ações para reverter essa situação. Segundo Andrade et al. (2020), foram realizadas com êxito, atividades voltadas para o público feminino em escolas públicas de Ensino Médio. 
De Oliveira et al. (2020a) em busca de promover a inovação e o empoderamento feminino, usaram a metodologia Design Thinking (DT), para ofertar oficinas que possibilitaram o debate sobre as dificuldades enfrentadas por mulheres na sociedade. Visando promover o protagonismo feminino, Pinheiro et al. (2020) relatam o uso do jogo de cartas Computasseia para promover conhecimentos referentes à História da Computação. O jogo possui o diferencial de abordar mulheres da computação.

O projeto Meninas Digitais do Vale, assemelha-se aos demais citados, por sua luta de instigar e apoiar a participação das mulheres na área da Ciência e Computação, de modo a realizar ações que também as tornem protagonistas. Portanto, o projeto adaptou suas atividades para a forma remota visando continuar alcançando seu público alvo.

\section{Ações realizadas em ambiente digital}

Nos anos de 2018 e 2019, o projeto Meninas Digitais do Vale realizou as ações de extensão com a plataforma Code.org e o jogo de cartas Computasseia na modalidade presencial através de visitas em escolas parceiras e turmas dos cursos de Computação da UFC - Campus Russas. Entretanto, essas ações ainda não foram realizadas de forma remota, pois não foi encontrado uma forma eficiente de acompanhar o rendimento dos estudantes. Novos cursos estão sendo propostos para atender ao público alvo do projeto no cenário remoto. Nesta seção são apresentadas as ações realizadas pelo projeto, visando apresentar a área de TI para o público, em sua maioria feminino.

\subsection{Cursos de longa duração}

Com o intuito de promover e incentivar a participação feminina nos cursos de TI, o projeto Meninas Digitais do Vale ofertou dois cursos de longa duração para estudantes do ensino fundamental e médio.

Prototipagem com gamificação "incentivando a criação de soluções digitais para os problemas oriundos do novo coronavírus": disponibilizado no formato online, dividido em oito semanas com carga horária de 80 horas. A metodologia adotada para a criação das soluções pelos estudantes foi a DT e foi utilizada a plataforma Classcraft $^{(1)}$ (Figura 1.a) como ambiente de ensino. Classcraft é uma plataforma de ensino gamificado que utiliza estratégias que permitem tornar a aprendizagem motivadora no ambiente escolar. Três bolsistas do projeto Meninas Digitais do Vale conduziram o curso sob orientação de duas docentes. Participaram 17 estudantes do $1^{\circ}$ ano e $3^{\circ}$ ano do ensino médio. Os estudantes foram divididos em três equipes com as temáticas: fome e erradicação da pobreza, saúde e bem-estar e economia sustentável. Etapas da metodologia utilizada: empatia, definição, idealização e prototipação (baixa e alta fidelidade). Todo material necessário para condução do curso foi disponibilizado na plataforma de ensino remoto e estão disponíveis no repositório do projeto $^{(2)}$. A análise dos resultados obtidos neste curso está descrita na Seção 4.

Iniciação à Lógica de Programação usando Scratch: o Scratch ${ }^{(3)}$ é uma linguagem de programação que utiliza blocos para estimular o ensino em Lógica de Programação. O curso teve duração de cinco semanas, consistia na resolução de desafios semanais, com teoria e prática, desde os princípios básicos da Lógica de Programação até o desenvolvimento de animações usando a ferramenta Scratch em sua modalidade 
website (Figura 1.b). O curso foi conduzido na plataforma gamificada Classcraft. A utilização dessa plataforma visou uma aprendizagem que incentiva a autonomia dos participantes. Vídeos e materiais explicativos foram produzidos previamente e disponibilizados na plataforma de ensino remoto, como também no repositório do projeto $^{(2)}$. Participaram do curso 15 estudantes do ensino fundamental e do primeiro ano do ensino médio de um colégio parceiro do projeto e pessoas oriundas da divulgação nas redes sociais. A análise dos resultados obtidos neste curso está descrita na Seção 4.

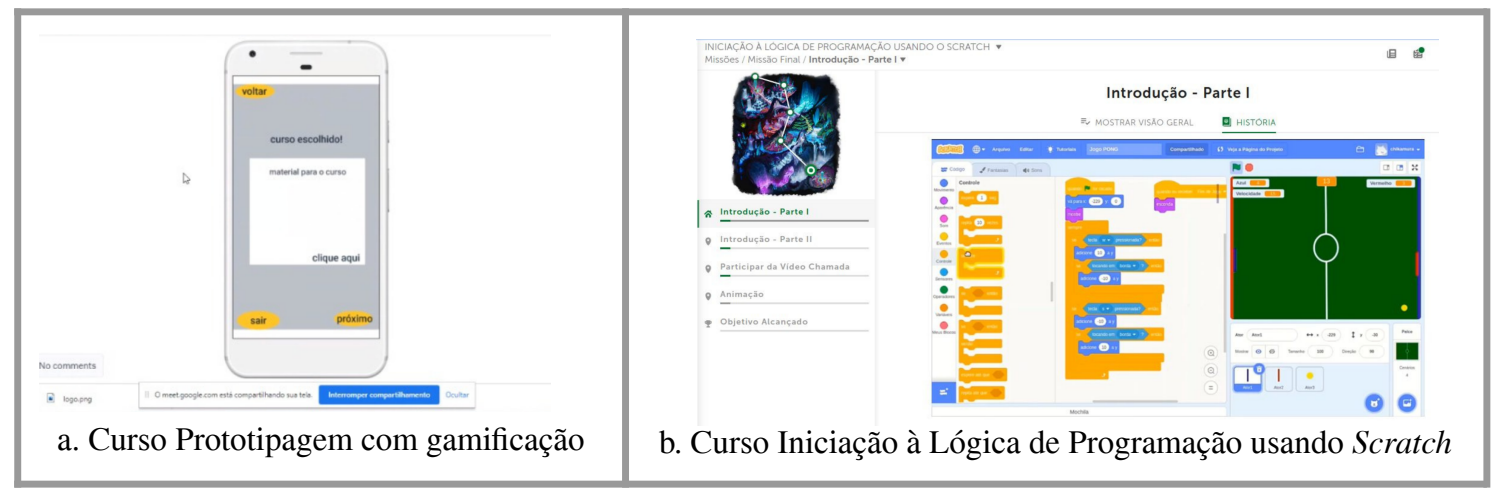

Figura 1. Cursos de longa duração

\subsection{Cursos de curta duração}

O projeto realizou três cursos online de curta duração. Por meio de enquetes feitas no Instagram do projeto, o público presente nas redes sociais do projeto, composto por estudantes universitários, professores e estudantes da educação básica, demonstrou interesse nos cursos de $\mathrm{Canva}^{(4)}$, Trello ${ }^{(5)} e \mathrm{Miro}^{(6)}$. As inscrições foram feitas através de um formulário no Google Forms ${ }^{(7)}$, contemplando universitários e pessoas que apresentavam interesse em participar. Os cursos foram realizados na plataforma Google $M e t^{(8)}$ com duração de duas horas e com direito a um certificado.

Canva: é uma plataforma de design gráfico que permite aos usuários criar gráficos de mídia social, apresentações, pôsteres e outros conteúdos visuais. Está disponível online e em dispositivos móveis e integra milhões de imagens, fontes, modelos e ilustrações. O curso Canva foi o primeiro a ser ofertado, houve duas turmas, a primeira obteve 147 inscrições e a segunda 43. Inicialmente foi explicado sobre o que é a plataforma, o que ela permite criar, seu funcionamento e ao final foi pedido para que os participantes criassem suas próprias artes.

Trello: é uma ferramenta de gerenciamento de projetos de forma colaborativa, muito utilizada para organização pessoal ou de grupo, na qual disponibiliza quadros para divisão de tarefas, auxiliando as pessoas a gerenciar suas atividades de forma gratuita e online, disponível para computadores e celulares. O curso Trello foi o segundo a ser ofertado, obteve 118 inscrições, a turma foi dividida em duas e em dois dias diferentes. Como prática final do curso ofertado, foi solicitado aos participantes a criação de quadros utilizando a ferramenta.

Miro: é uma plataforma de quadro colaborativo online que permite que equipes distribuídas trabalhem efetivamente em conjunto. Fornece funcionalidade básica para smartphones (Android e IOS) e um aplicativo completo na web. $\mathrm{O}$ curso miro foi o 
terceiro a ser ofertado, obteve 40 inscrições e teve uma turma. Ao final, foi solicitado aos alunos a realização do cadastro na plataforma e criação de um brainstorming ou um mapa mental para praticar os conteúdos abordados durante o curso.

\subsection{Promoção de Evento: Campanha Outubro Rosa Sob Novos Olhares}

O projeto Meninas Digitais do Vale em parceria com outros projetos da Universidade Federal do Ceará - Campus Russas, promoveram no mês de outubro de 2020 a Campanha Outubro Rosa Sob Novos Olhares. O evento teve como objetivo levar ao público informações sobre a prevenção ao câncer de mama e o cotidiano feminino. Esse evento foi gratuito e aberto ao público em geral. Foram realizadas mesas-redondas em formato de lives com profissionais renomados abordando temas importantes do universo feminino, como a saúde sexual e reprodutiva da mulher, a prevenção ao câncer de mama e a outros tipos de câncer, e a violência contra a mulher.

\subsection{Produção de conteúdos temáticos para o Instagram}

Antes da pandemia o Instagram do projeto $^{\left({ }^{(9)}\right.}$ não era constantemente utilizado, servia para registro e divulgação das ações do projeto realizadas durante o semestre. No período de pandemia, visando reinventar as ações e aumentar o engajamento dos seguidores, atingindo a um público diverso no Instagram do projeto Meninas Digitais do Vale, foi desenvolvido um calendário contemplando a divulgação de diferentes temas e assuntos para todos os públicos. Os principais temas divulgados foram:

Mulheres na Computação: os cursos de tecnologias apresentam baixo índice de pessoas do sexo feminino, necessitando de mais incentivo para as mulheres na área da Computação (Flôr et al., 2020). O intuito da postagem sobre Mulheres na Computação é divulgar biografias e feitos de mulheres que contribuíram para o desenvolvimento da Ciência da Computação e da TI. Dessa forma, espera-se que o público tenha conhecimento das contribuições dessas mulheres para a história e que elas sirvam de fonte de inspiração. Dentre as mulheres na Computação apresentadas, teve: Ada Lovelace, Katie Bouman, Margaret Hamilton, Susan Wojcicki e Hedy Lamarr.

Mulheres que inspiram: tendo em vista que na UFC encontram-se profissionais dedicadas e que servem como motivação para as pessoas, foi sugerido a postagem sobre Mulheres que inspiram no campus Russas. O objetivo é divulgar as biografias das servidoras e professoras, ou seja, uma forma de mostrar exemplos de mulheres capacitadas que fazem parte do campus.

Homenageadas do campus: no meio acadêmico existem muitas estudantes com histórias que se destacam. O projeto buscou uma forma de homenagear essas estudantes que encorajam as demais a se manterem firmes em suas jornadas, através do uso de um formulário, divulgado na rede social do projeto, o público pôde indicar uma dessas estudantes que os inspiraram para que recebessem uma singela homenagem, com uma publicação sobre sua história, ações e motivos que as fazem ser referência.

Dicas sobre profissões de TI: para as pessoas com curiosidade em conhecer as diversas profissões de TI, as integrantes do projeto passaram a divulgar dicas de profissão de TI. Assim, o público do projeto ganha conhecimento e pode despertar o 
interesse/afinidade com alguma dessas profissões. Dentre as apresentadas, teve: Analista de Sistemas, Desenvolvedor(a), Designer e Cientista de Dados.

Dicas de escrita científica: visando auxiliar e incentivar a continuidade de pesquisas e produção de artigos e resumos científicos, foram publicadas Dicas para escrita de artigos científicos, tendo como base as lições aprendidas das integrantes do projeto. As dicas abordaram: resumo estruturado, introdução, background e trabalhos relacionados e desenvolvimento segundo a norma técnica de escrita.

Conceitos de programação com Computação Desplugada: pensando em estimular o conhecimento em programação, o projeto publicou dicas que enriquecem o entendimento sobre o assunto. Dentro desse tópico, foram exemplificadas atividades envolvendo Computação Desplugada que possui uma didática simples e interativa sobre conceitos computacionais sem a necessidade de um computador. As atividades envolveram Código Binário, Criptografia e Representação de Imagens.

Dicas sobre técnicas de IHC: no intuito de mostrar diferentes técnicas e ampliar as possibilidades de uso de IHC, o projeto Meninas Digitais do Vale passou a apresentar Dicas de utilização de técnicas de IHC e suas principais características. As técnicas de IHC abordadas foram: Prototipação, Avaliação Heurística, Teste de Usabilidade (UX), Card Sorting, Personas, e Grupo de Foco.

WebSérie: com o objetivo de motivar e tentar sanar as dúvidas de estudantes de TI acerca das diferentes áreas de atuação, o projeto criou uma WebSérie chamada " $E$ você mulher de TI, qual carreira seguir?". Foram produzidos vídeos com as convidadas respondendo uma série de perguntas sobre a sua jornada, os desafios enfrentados e como a mulher é vista na área de TI. Os vídeos mostram a trajetória de alunas egressas dos cursos de tecnologia da UFC - Campus Russas, seja no mercado de trabalho ou em uma pós-graduação. Os vídeos estão disponíveis no Instagram do projeto ${ }^{(\mathbf{9})}$. Até o momento foram ao ar quatro episódios, somando um total de 1110 visualizações.

\section{Feedback de participantes sobre as ações realizadas}

As próximas seções abordam o feedback de participantes das ações do projeto. Os resultados obtidos foram coletados por meio de questionários ${ }^{(2)}$ e enquetes no Instagram e foram analisadas por meio da análise qualitativa e quantitativa.

\subsection{Cursos de curta duração}

Uma análise qualitativa foi realizada com as respostas de dez participantes que se disponibilizaram a responder um questionário online composto por oito perguntas que avaliava os minicursos. Todas as questões do questionário estão disponíveis no repositório do projeto. Nessa seção, duas questões são apresentadas. Essas questões são importantes para a compreensão da experiência das participantes nos minicursos e mostram informações sobre as motivações, as ideias e as atitudes dessas participantes.

Os resultados obtidos para a questão que investigou os aprendizados adquiridos com os minicursos oferecidos pelo projeto são apresentados na Tabela 1. Com base nos resultados, é possível observar que os minicursos estimularam a criatividade e auxiliaram na utilização das plataformas. A Tabela 2 mostra os resultados obtidos para a questão que buscou obter sugestões de melhorias para os minicursos. 
Tabela 1. Percepções sobre os cursos de curta duração

\begin{tabular}{|l|l|}
\hline \multicolumn{2}{|c|}{ Questão: “Em que os treinamentos (minicursos) do nosso projeto mais contribuiu na sua formação?” } \\
\hline \multicolumn{1}{|c|}{ Percepção Identificada } & \multicolumn{1}{c|}{ Citação } \\
\hline Os cursos estimulam a criatividade & "(..) pude desenvolver artes mais criativas com o Canvas" - (P3) \\
\hline $\begin{array}{l}\text { Os treinamentos auxiliaram a } \\
\text { aprender a utilizar ferramenta a usar ferramentas que agregam no meu conhecimento } \\
\text { e utilização no dia a dia" - (P4) } \\
\text { "Aprendi a usar uma plataforma que é muito interessante, além } \\
\text { de bem útil no meu meio estudantil, principalmente que estamos } \\
\text { em EAD (...)" - (P8) }\end{array}$ \\
\hline
\end{tabular}

Analisado a Tabela 2, é possível observar algumas sugestões: criação de materiais de apoio para auxiliar na aprendizagem e cursos com maior duração.

Tabela 2. Percepções sobre os cursos de curta duração

\begin{tabular}{|l|l|}
\hline \multicolumn{2}{|l|}{ Questão: "Quais melhorias/sugestões você considera importante para novas edições do curso que você } \\
participou?"
\end{tabular}

A análise quantitativa foi feita sobre os dados coletados através de enquetes no Instagram do projeto e por um questionário ${ }^{(2)}$ enviado para os e-mails de participantes dos minicursos, obtendo nove respostas. Como a análise objetivou avaliar aspectos como a expectativa, satisfação e qualidade, foram destacadas nesse artigo as questões que investigavam esses aspectos, sendo possível obter informações relevantes sobre a experiência das participantes. A seguir estão descritos alguns resultados adquiridos.

Sobre a questão "Os treinamentos realizados atenderam suas expectativas? (Duração, conteúdo, forma de ensino)", 88,9\% indicaram concordância (sim) e 11,1\% indicaram "parcialmente". Ao analisar as questões "O treinamento estava bem estruturado, lhe fornecendo uma boa experiência?" e "Você teve um bom nível de aproveitamento das informações passadas, conseguindo usar a plataforma de maneira satisfatória?" obteve $100 \%$ de concordância. Deste modo, demonstra-se que a maioria das participantes teve uma boa experiência com o curso e as expectativas atendidas.

\subsection{Curso de longa duração: Prototipagem com gamificação}

No curso de Prototipagem com gamificação foi realizada uma análise qualitativa com os dados obtidos através de entrevistas realizadas pelas tutoras com os participantes do curso sobre a percepções de utilização da plataforma Classcraft. Os resultados obtidos para a questão que buscou identificar a utilidade da plataforma para o processo de ensino e aprendizagem dos alunos são apresentados na Tabela 3. Em relação a essa questão, os participantes consideram que a organização da plataforma auxiliou no aprendizado e que promove um ambiente que estimula o aprendizado.

A questão apresentada na Tabela 4 visou identificar quais recursos do Classcraft contribuíram para o engajamento dos participantes durante o curso. Observou-se que os 
participantes consideram que a organização das missões e ordem dos elementos na plataforma contribuíram para o engajamento.

Tabela 3. Percepções sobre o curso de prototipação

\begin{tabular}{|c|c|}
\hline \multicolumn{2}{|c|}{ Questão: “Em sua opinião, com o uso da plataforma Classcraft para o ensino, você acredita que } \\
aprendeu o conteúdo? Comente."
\end{tabular}

Intuindo avaliar a experiência dos estudantes do curso de prototipação foi utilizado o questionário GEQ (Game Experience Questionnaire) ${ }^{(2)}$ (IJsselsteijn et al., 2013) que é composto por um conjunto de dimensões, sendo algumas delas: diversão, competência, tensão e fluxo. Alguns resultados estão descritos a seguir.

Ao analisar a afirmativa: "Eu me senti tenso", observou-se que 63,6\% dos participantes responderam "de modo nenhum" e 18,2\% dos participantes consideram "moderadamente". Na afirmativa: "Eu senti que estava aprendendo", 54,5\% dos participantes concordaram bastante e $45,5 \%$ concordaram extremamente. Na afirmativa: "Eu atingi as metas", contabilizou que 9,1\% dos participantes consideram que não atingiram as metas de modo nenhum e $9 \%$ dos participantes atingiram as metas ligeiramente. Já a afirmativa: "Eu achei difícil", 72,7\% dos participantes consideram de modo nenhum e 27,3\% ligeiramente. Além disso, na afirmativa: "Pensei em outras coisas durante o jogo", contabilizou-se que $36,4 \%$ dos participantes consideram que de modo nenhum e $36,4 \%$ ligeiramente. Portanto, nota-se que os estudantes acharam as atividades desafiadoras, mas não as consideram difíceis, além de terem sentido que estavam aprendendo o conteúdo. Isso mostra que os desafios propostos fizeram com que os estudantes tivessem a sensação de aprendizado.

Tabela 4. Percepções sobre o curso de prototipação

\begin{tabular}{|c|c|}
\hline \multicolumn{2}{|c|}{$\begin{array}{c}\text { Questão: "Em sua opinião, quais recursos do Classcraft contribuíram para o engajamento ao longo do } \\
\text { curso?" }\end{array}$} \\
\hline & \\
\hline $\begin{array}{l}\text { Organização das missões } \\
\text { contribuiu para o } \\
\text { engajamento do curso }\end{array}$ & $\begin{array}{l}\text { "(...) os recursos que contribuíram para o engajamento foi essa } \\
\text { questão das missões serem separadas em baixo e alto nível" - (P2) } \\
\text { "(...) é muito interessante no Classcraft que ele fica separado, primeiro } \\
\text { que tem a parte da explicação né e depois a da tarefa, (...) muito legal } \\
\text { a parte do mapa e as tarefas ficam separadinhas" - (P7) }\end{array}$ \\
\hline $\begin{array}{l}\text { Pontuação das tarefas } \\
\text { auxiliou no engajamento do } \\
\text { curso }\end{array}$ & $\begin{array}{l}\text { "(...) foi mesmo um sistema de level e de ponto pela atividade também } \\
\text { ajudou demais tipo contribuir para as atividades (...)" - (P8) } \\
\text { "Acho que a parte dos pontos, quando você ver que vai ganhando } \\
\text { pontos você vai ficando mais engajado e vai ficando mais, como posso } \\
\text { dizer? com mais vontade de aprender." - (P10) }\end{array}$ \\
\hline
\end{tabular}

\subsection{Curso de longa duração: Iniciação à Lógica de Programação Usando Scratch}

Para avaliar a experiência das participantes do curso de lógica de programação com Scratch foi aplicado um questionário ${ }^{(2)}$ com a finalidade de avaliar a satisfação e 
possíveis dificuldades, coletando a percepção das três estudantes do gênero feminino que concluíram o curso.

Em relação à questão "Você achou as atividades do curso difíceis?" percebeu-se que todas as participantes discordaram que as atividades do curso são difíceis. Assim como, ao analisar as questões " $O$ curso estava bem estruturado, lhe fornecendo uma boa experiência?" e "você se sentiu satisfeito em realizar as atividades propostas no curso?" todas as participantes concordam. Em suma, os dados demonstram que o curso promoveu boa experiência e satisfação aos participantes.

\section{Lições Aprendidas}

Diante da boa experiência proporcionada pelos cursos de curta duração, os resultados apresentam necessidade de aumentar a duração das ações, com isso, os cursos serão em dois dias com duração de quatro horas e será fornecido um material de apoio ao final de cada dia do curso. A produção de conteúdo temático para o Instagram promoveu o aumento de seguidores nas redes sociais do projeto e do engajamento nas publicações. Diante disso, o projeto recebeu sete prêmios na premiação Top Awards promovido pelo time organizacional publicitário da UFC - Campus Russas obtendo o primeiro lugar nas categorias: "Melhor design gráfico", "Melhor identidade visual" e "Presença no instagram" e o segundo lugar nas categorias "Melhores edições audiovisuais", "Melhor produção de conteúdo", "Canal revelação" e "Melhor composição fotográfica".

No curso de lógica de programação com Scratch, as participantes relataram como um ponto forte a flexibilidade do ensino remoto, porém informaram que a plataforma web dificultou a realização das tarefas. Como lição aprendida, o curso utilizará a versão do Scratch para mobile, pois apresenta facilidade de uso. Dessa forma, espera-se promover a inclusão dos participantes que não têm acesso ao computador. Ademais, no curso de prototipação com gamificação os participantes relataram que os materiais de apoio disponibilizados pelas tutoras na plataforma Classcraft os auxiliaram no aprendizado dos conteúdos. Além disso, em virtude das sugestões de melhoria propostas pelos participantes, observou-se a necessidade de adicionar uma pontuação maior para tarefas do curso para possibilitar maior personalização dos avatares, bem como a criação de tutoriais de iniciação e utilização da plataforma.

\section{Considerações Finais e Trabalhos Futuros}

Por meio da pesquisa realizada pode-se perceber que mesmo com os impactos causados pela pandemia do COVID-19, o projeto Meninas Digitais do Vale conseguiu continuar suas atividades de forma remota, mantendo a qualidade das ações realizadas e procurando formas de produção de conteúdo mediante ao cenário atual. Percebe-se também por meio da análise dos resultados a aceitação do público com a forma de condução das ações remotas e com os conteúdos apresentados. Logo, é possível identificar o impacto positivo causado por essas ações. As ações realizadas na mobilidade remota em comparação às ações presenciais abrangeram um público maior e diverso, composto por professores e estudantes do ensino fundamental ao superior.

Conforme as expectativas do cenário atual, o projeto tem como trabalhos futuros continuar realizando ações voltadas para o público feminino e procurar mais formas de 
engajar as meninas em atividades remotas, compreendendo sempre a situação atual e buscando estratégias para moldar esse cenário. Também pretende-se realizar rodas de conversas para promover maior integração entre as alunas, assim como novos cursos e treinamentos que contemplem outras áreas da TI para que cada vez mais meninas se sintam encorajadas a participarem das ações realizadas pelo projeto.

\section{Referências}

Andrade, L. M. M. D. S., de Oliveira Santos, S., Gomes, M. R., Rodrigues, A. A. M., de Miranda, A. C., Torres, L. L. S. and Barbosa, C. S. (2020). Ações Iniciais do Projeto de Extensão Garotas Tech dos Sertões de Crateús para Captação e Retenção de Meninas aos Cursos de TI da UFC-Crateús. In Anais do XIV WIT, p. 169-173. SBC.

BRASIL (2020). Portaria No 343, de 17 de março de 2020. Dispõe sobre a substituição das aulas presenciais por aulas em meios digitais enquanto durar a situação de pandemia do Novo Coronavírus - COVID-19.

de Oliveira, L. M. C., Oliveira, R. A., and Marques, A. B. (2020a). Design Thinking: oficinas para inovação e empoderamento feminino. In Anais do XIV Women in Information Technology, p. 204-208. SBC.

de Oliveira, P. A., Maciel, A. and Souza, G. F. (2020b). Projeto Meninas na Computação-UNIFAP: relato de experiência e desafios. In Anais do XIV Women in Information Technology, p. 264-268. SBC.

Flôr, D. E., da Cruz, E. H. M., Possebom, A. T., Junior, C. R. B., Hübner, R., Gonçalves, P. C., da Silva, C. A. and Aylon, L. B. R. (2020). MannaAcademy: impulsionando o protagonismo feminino através de uma rede interinstitucional de extensão universitária. In Anais do XXVIII Workshop sobre Educação em Computação, p. 26-30. SBC.

Frigo, L. B., Moro, F. F., Padilha, R. O. and Pozzebon, E. (2020). Meninas em Ação: Atividades Inspiradoras para Projetos Parceiros do Programa Meninas Digitais. In Anais do XIV Women in Information Technology, p. 60-69. SBC.

IJsselsteijn, W. A., de Kort, Y. A. and Poels, K. (2013). The Game Experience Questionnaire. Eindhoven: Technische Universiteit Eindhoven, v. 46, n. 1.

Maciel, C., and Bim, S. A. (2016). Programa Meninas Digitais-ações para divulgar a Computação para meninas do ensino médio. Anais do Computer on the Beach, p. 327-336.

Ministério da Saúde. (2020). Portaria $n^{\circ} 188$, de 3 de fevereiro de 2020. Declara Emergência em Saúde Pública de importância Nacional (ESPIN) em decorrência da Infecção Humana pelo novo Coronavírus (2019-nCoV). Diário Oficial da União.

Nobre, C. D. F. C. and Guerreiro, M. Â. N. (2020). Ensinar e Aprender em Tempos de Pandemia Covid-19. Saberes Plurais: Educação na Saúde, v. 4, n. 2, p. 6-10.

Pinheiro, V. M., Alencar, A. I., Branco, K. C., Fiori, M. V., and Marques, A. B. (2020). Ensinando História da Computação com foco no protagonismo das mulheres com jogo Computasseia: Um relato de experiência com ingressantes de Engenharia de Software e Ciência da Computação. In Anais do XIV WIT, p. 214-218. SBC.

\section{Links Citados no Artigo}

${ }^{(1)}$ https://www.classcraft.com/pt/; ${ }^{(2)}$ https://bit.ly/Repositorio_MDV; ${ }^{(3)}$ https://scratch.mit.edu/;

${ }^{(4)}$ https://www.canva.com; ${ }^{(5)}$ https://trello.com/; ${ }^{(6)} \mathrm{https} / / / \mathrm{miro} . c o m /$;

${ }^{(7)}$ https://docs.google.com/forms/; ${ }^{(8)}$ https://meet.google.com/;

${ }^{(9)}$ https://www.instagram.com/meninasdigitaisdovale/; ${ }^{(10)}$ https://forms.gle/42P6iZ4UGNkKp13z6 